\title{
Avaliação da RelaÇão entre O déficit de ATENÇÃo E O deSEM PENHO gRAFo-MOtor em ESTUdANTES COM SíndRome de Down
}

\author{
EVALUATION OF THE RELATIONSHIP BETWEEN ATTENTION-DEFICIT DISORDER AND \\ THE DEVELOPMENT OF GRAPHOMOTOR SKILLS IN STUDENTS WITH DOWN \\ SYNDROME
}

\author{
Luciel MACÊDO ${ }^{1}$ \\ Iris LIMA ${ }^{2}$ \\ Fabrício CARDOSO ${ }^{3}$ \\ Heron BERESFORD ${ }^{4}$
}

\begin{abstract}
RESU M 0: este estudo teve como objetivo avaliar a relação existente entre o déficit de atenção e o desempenho de uma conduta grafo-motora necessária à aquisição da linguagem lecto-escrita em cinco estudantes com Síndrome de Down, de ambos os sexos, com idade compreendida entre 17 e 21 anos, matriculados na Escola Estadual Presidente Costa e Silva em Belém-PA. A metodologia de trabalho foi própria de uma avaliação de contexto que permitiu elaborar um estudo descritivo e correlacional. Como estratégia metodológica foram realizados dois testes: um de Processamento Mental para avaliar o tempo de reação a um estímulo programado e outro de avaliação das variáveis relacionadas à conduta grafo - motora. Os resultados indicaram uma estreita relação entre as duas variáveis pesquisadas, ou seja, estudantes que apresentaram um longo tempo de reação alcançaram um baixo escore no teste grafo motor e aqueles que tiveram rápido tempo de reação obtiveram um alto escore no teste grafo-motor.
\end{abstract}

PALAVRAS-CHAVE: avaliação; síndrome de down; déficit de atenção; conduta grafo-motora.

\begin{abstract}
: this study aimed to evaluate the relationship between attention-deficit disorder and the development of graphomotor skills in language acquisition processes related to reading and writing readiness skills. The participants of the study were five students with Down syndrome, both sexes, aged 17-21, enrolled at Presidente Costa e Silva School, Belém-PA. The working methodology was a contextual evaluation within a descriptive and correlational study framework. As a methodological strategy, two tests were performed: a mental concentration processing test aimed to evaluate the reaction time to an input stimulus and a Graphomotor Developmental Test was designed to analyze the variables involved in the development of the graphomotor skills. The results indicated that there was a strict relationship between RT (reaction time) and the scores on the graphomotor tests. A low RT implied a low score on the graphomotor developmental test while a high RT implied a high score on the graphomotor developmental test.
\end{abstract}

KEYWORDS: Special Education; Evaluation; Down Syndrome; Attention-deficit disorder; Development of Graphomotor Skills.

\footnotetext{
${ }^{1}$ Professor de Biologia do ensino médio da rede pública e privada em Belém-Pa, coordenador do laboratório multidisciplinar da escola Estadual Orlando Bitar, aluno do curso de mestrado em Ciência da Motricidade Humana UCB-RJ lucielmacedo@yahoo.com.br

2 Mestre em Ciência da Motricidade Humana/ Universidade Castelo Branco/ RJ - irislimaucb@yahoo.com.br ${ }^{3}$ Mestre em Ciência da Motricidade Humana/ Universidade Castelo Branco/ RJ - fbc@bmrio.com.br

${ }^{4}$ Doutor em Filosofia - UGF; Prof. Titular do Programa de Pós-Graduação Stricto Senso em Ciência da Motricidade Humana da Universidade Castelo Branco - UCB - RJ; Prof. Adjunto da Universidade do Estado do Rio de Janeiro - UERJ . Coordenador de Pesquisas no LABFILC - Laboratório de Temas Filosóficos em Conhecimento A plicado- heronb@terra.com.br
} 


\section{INTRODUÇÃo}

A problemática que trata de vários aspectos relativos à dificuldade da aprendizagem de al unos com Síndrome de Down (SD) é um assunto bastante complexo e polêmico. Durante décadas a avaliação sobre o desenvolvimento cognitivo dessas pessoas ficou marcada por entendimentos estereotipados que hoje, com o avanço dos estudos, começam a ser revistos. Assim, novas perspectivas de interpretação e novas tomadas de decisão, surgem como formas alternativas de intervenção, contribuindo para potencializar tal desenvolvimento não só em termos de domínio das habilidades intelectuais como, também, em um espectro mais amplo de desenvolvimento humano.

Embora muitos acreditem queo processo deaprendizagem das pessoas com SD se dá apenas deforma mais lenta quea das outras pessoas, há, na verdade, segundo Wuo (2007), diferenças estruturais que tornam esse processo essencialmente diferente do das outras pessoas. Assim, vejamos.

Para Silva e Kleinhans (2006), as diferentes formas de lesões que acometem os indivíduos com SD influenciam o seu desenvolvimento e aprendizagem e, neste contexto, como bem frisa Schwartzman (2003), embora essas alterações biofísicas ocorram em vários sistemas do organismo, são particularmente frequentes e importantes as alterações no sistema nervoso central, determinantes das disfunções neurológicas que estão sempre presentes na SD, variando, entretanto, quanto às manifestações e intensidade das mesmas.

Schwartzman (2003) considera que a linguagem é a área na qual a criança com SD demonstra, em geral, os maiores atrasos, emitindo a primeira palavra, em média, aos 18 meses e, geralmente, pode compreender bem mais do que emitir. O autor destaca, porém, que, apesar de ter uma síndrome, a criança com SD será sempre singular, ou seja, o fato de existir um grupo de crianças com características físicas, cognitivas e de linguagem semelhantes não significa que elas sejam igualmente competentes ou incompetentes para dialogar, pois, se cada criança se desenvolve em um ambiente diferente, não poderemos encontrar uma criança idêntica à outra, se analisarmos profundamente sua linguagem.

A aprendizagem da linguagem se dá por meio de modificações estruturais e funcionais do Sistema Nervoso Central (SNC), principalmente nas áreas da linguagem, das gnosias, das praxias, da atenção e da memória e, para que este processo se estabeleça corretamente, é necessário que as interações entre as diversas áreas corticais e delas com outros níveis do SN C sejam efetivas. Desta forma, alterações que ocorram neste sistema poderão comprometer consideravel mente a aprendizagem e, como sabemos, estas al terações podem ser observadas em crianças com SD. As alterações difusas, porém constantes das áreas sensoriais, associativas e pré-frontais do córtex cerebral são elementos primordiais do déficit cognitivo na SD, que se relaciona de forma mais marcante às competências linguísticas. (ROTA; OHLWEILER; RIESGO, 2006). 
A lém das alterações difusas do córtex cerebral, outras áreas do SNC podem estar comprometidas. Isto pode ser verificado em Flórez eTroncoso (1997), quando relatam que todos os neurônios formados são afetados na maneira como se organizam em diversas áreas do sistema nervoso e não só há alterações na estrutura formada pelas redes neuronais, mas também nos processos funcionais da comunicação de um com o outro. Destaca-se, ainda, a influência que essas alterações podem exercer sobre o desenvolvimento inicial nos circuitos cerebrais, afetando a instalação e as consolidações das conexões de redes nervosas necessárias para estabelecer os mecanismos da atenção, da memória, da capacidade de correlação e análise e do pensamento abstrato.

Os estudos com crianças com SD, relatados por Schwartzman (2003), descrevem alterações na árvore dendrítica e, em particular, nas espinhas dendríticas que, como se sabe, são estruturas pós-sinápticas essenciais dos circuitos neurais. De acordo com Casarin (2003), informações baseadas em estudos de Dunst (1988), Green et al. (1989), Becker et al. (1991) e Flores (1995), revelam que tais alterações nas ramificações dendríticas, principalmente, no lado frontal, ea redução na formação si náptica seriam a causa do déficit de atenção observado em crianças com a SD.

O déficit de atenção, segundo Casarin (2003), pode interferir no desenvolvimento dessas pessoas, pois dificulta a iniciação, a organização e a manutenção do envolvimento na realização de determinadas tarefas, como por exemplo, condutas grafo-motoras, necessárias para a aquisição da linguagem escrita.

Gomes (2005) considera a atenção indispensável à interação eficaz do indivíduo com o seu ambiente, pois, por meio desta função mental pode-se selecionar qual estímulo será analisado em detalhes e qual será levado em consideração para guiar nosso comportamento, sendo, portanto, a base para a organização dos processos mentais, conferindo a estes diretividade, seletividade e estabilidade. A diretividade será responsável pela orientação em busca do estímulo; a seletividade permitirá concentrar o foco da atividade nos estímulos significativos; e a estabilidade permitirá a adoção de uma postura facilitadora à recepção do estímulo, ou seja, nesteprocesso, o sistema nervoso écapaz de manter um contato seletivo com as informações que chegam por meio dos órgãos sensoriais, dirigindo a atenção para aqueles que são comportamentalmente relevantes e garantindo uma interação eficaz com o meio (BRANDÃO, 1995).

Dulcan e Benson (1997) esclarecem que o estado atentivo contribui decisivamente para as al terações que se operam na organização cognitiva e que, em última instância, são responsáveis pela aprendizagem e pela memorização do conteúdo apreendido. A atenção representa, pois, condição inicial e decisiva para as funções cognitivas em sua totalidade, particularmente para o processo de aprendizagem e de memorização. Indivíduos com déficit de atenção, em geral, não apresentam bom desempenho nestes processos, ressaltam os autores. 
Segundo Mills (2003), crianças com SD apresentam algumas deficiências que interferem nos diversos processos do desenvolvimento humano em geral, destacando as dificuldades nas tomadas de decisões; na sel eção e eliminação de determinadas fontes informativas e no bloqueio de funções receptivas como a atenção e percepção.

Dessa forma, é razoável supor-se que a dificuldade na coleta, na análise e na seleção de novas informações compromete bastante o aprendizado geral de crianças com SD, podendo vir a interferir, no processo de aquisição da linguagem lecto escrita de forma muito particular.

A partir da problemática anteriormente apresentada e justificada, evidencia-se que o objetivo deste estudo foi avaliar a correlação entre o estado de atenção e o desempenho de conduta grafo-motora em alunos com Síndrome de Down, de ambos os sexos e com idade compreendida entre 17 a 21 anos. Isto com o propósito de que os resultados desta avaliação sirvam para o planejamento de futuras intervenções relevantes ou com um impacto social mente positivo para os entes pesquisados.

\section{METOdOLOGIA}

\section{TIPOLOGIA E MÉTODO DO ESTUDO}

Nesta pesquisa se utilizou o método de uma avaliação de contexto que permitiu elaborar um estudo descritivo e correlacional acerca do estado de atenção com o desempenho de conduta grafo-motora em alunos com Síndrome de Down, de ambos os sexos e com idade compreendida entre 17 a 21 anos.

\section{UNIVERSO}

Este estudo foi realizado com cinco estudantes com SD, de ambos os sexos e idade compreendida entre 17 e 21 anos, matriculados na segunda etapa do ensino fundamental, da Escola Estadual de Ensino Fundamental e Médio, Presidente Costa e Silva em Belém-PA que, de acordo com a Teoria da A mostragem formulada por Cochran (1956), formou um grupo denominado censo, visto que todos os alunos com SD, da referida escola, foram submetidos aos procedimentos de avaliação.

\section{Estratégias METOdOLÓgICAS}

Todos os estudantes do grupo censo foram submetidos, na mesma Escola em que os referidos al unos frequentam, deforma individual e nos horários normais de aula, a dois testes: 
a) Teste de A valiação de conduta grafo-motora

N este teste foram avaliadas as variáveis relacionadas à conduta grafo - motora, ou seja, velocidade, coordenação de agilidade, equilíbrio, coordenação bilateral, força, motricidade global, coordenação dos membros superiores, motricidade composta (velocidade de reação, viso-motricidade, dextralidade) e motricidade fina (proficiência motora).

Cada estudante, de forma individual, foi orientado a escrever uma palavra que Ihe era mostrada no quadro pelo professor. Em seguida, sob a mesma orientação eram solicitados a escrever seu nome, onde, para efeito de escore, cada variável acima era observada e pontuada num escore de 0 a 10 pontos, tendo este teste um escore máximo de 100 pontos (SILVA; RABELLO, 2004).

b) Teste de Processamento $M$ ental

No testedeprocessamento mental, teve-seo intuito deavaliar "o tempo que decorre da apresentação de um estimulo não-antecipado ao inicio da resposta" (SCHMIDT; WRISBERG, 2001). O tempo de reação ao estímulo foi medido por meio do teste de dupla escolha, elaborado por Silva (2004).

Para a execução deste teste, foi solicitado a cada aluno que se posicionasse sentado frente a um computador. Na tela do aparelho, foram apresentados três quadrados: um localizado no centro superior da tela, para acender como luz de alerta; os outros dois quadrados apareciam, cada um, num canto inferior da tela, funcionando como luz de estimulo na direita ou na esquerda, de forma aleatória.

O tempo de alerta foi de quatro segundos para o inicio do estímulo. A pós o estímulo, o aluno deveria executar uma atividade motora que consistia em apertar uma tecla correspondente a uma letra, previamente estabel ecida, ou seja, a tecla com a letra A, caso o estímulo aparecesse no quadrado do lado esquerdo e a tecla com a letra L, caso o estímulo aparecesse no lado direito.

Solicitou-se aos alunos que realizassem dez tentativas, sendo, para cada uma, medido o tempo de resposta, assim considerado: para a luz do lado direito, a resposta deveria ocorrer do lado direito; para a luz do lado esquerdo, a resposta deveria ocorrer do lado esquerdo.

Com os resultados dos dois testes especificados, foi avaliada a correlação entre o estado de atenção e o desempenho da conduta grafo-motora. Para isto, os valores do Teste de Processamento Mental foram convertidos em Percentual (variando de $0 \%$ a $100 \%$ ), calculando-se, posteriormente, o valor Estimado Máximo (9.4263) e o valor Estimado Mínimo (0.7717), a partir da estimativa do interval o de confiança IC 99\%, baseados na distribuição t deStudent (AYRES, 2008). 
Em seguida, a Escala Percentual do Teste de Processamento Mental foi Invertida, ou seja, o valor $0 \%$ passou a valor $100 \%$; o valor $10 \%$ passou a valer $90 \%$; o valor $20 \%$ passou a valer $80 \%$ assim, todos os valores foram convertidos.

\section{Ética da Pesquisa}

O projeto desta pesquisa foi submetido ao ComitêdeÉtica em Pesquisa envolvendo Seres Humanos da Universidade Castelo Branco (UCB/ RJ) e aprovada sob protocolo no 0179/ 2008

A coleta de dados se deu conforme a resolução 196/ 96 do Conselho Nacional de Saúde do Ministério da Saúde. Assim, inicialmente foi solicitada a autorização dos pais ou responsáveis para que o estudo fosse desenvolvido, por meio de um Termo de Consentimento Livre e Esclarecido.

\section{A PRESENTAÇão e discussão dos Resultados}

A Tabela 1, a seguir, apresenta os resultados do teste Grafo-motor e do teste de Processamento Mental.

Tabela 1 - Avaliação do estado de atenção e do desempenho da conduta grafomotora $(n=5)$.

\begin{tabular}{lccc}
\hline & $\begin{array}{c}\text { Teste Grafo- } \\
\text { Motor }\end{array}$ & \multicolumn{2}{c}{ Teste de Processamento } \\
& Mental \\
\cline { 2 - 4 } Especificidades & $\begin{array}{c}\text { Escores } \\
\text { Primários }\end{array}$ & $\begin{array}{c}\text { Percentual } \\
\text { Invertido }\end{array}$ & $\begin{array}{c}\text { Escores } \\
\text { Primários }\end{array}$ \\
\hline Mínimo de pontos obtidos & 4.0 & 20.9 & 1.5 \\
Primeiro Quartil (25\%) & 19.0 & 38.3 & 2.9 \\
Mediana & 28.0 & 63.7 & 3.8 \\
Média Aritmética & 27.6 & 57.6 & 4.4 \\
Terceiro Quartil (75\%) & 43.0 & 74.5 & 6.1 \\
Máximo de pontos obtidos & 44.0 & 90.5 & 7.6 \\
Desvio Padrão & 16.9 & 27.9 & 2.5 \\
Coeficiente de Variação & $61.09 \%$ & $48.54 \%$ & $55.90 \%$ \\
\hline
\end{tabular}

Fonte: Dados da Pesquisa

O Teste Grafo-motor apresentou resultados variando entre 04 e 44 pontos. Os val ores que representam a tendência central da amostra são: mediana $=28$ e média $=27.6$. Este teste apresentou Coeficiente de Variação $=61.09 \%$. 


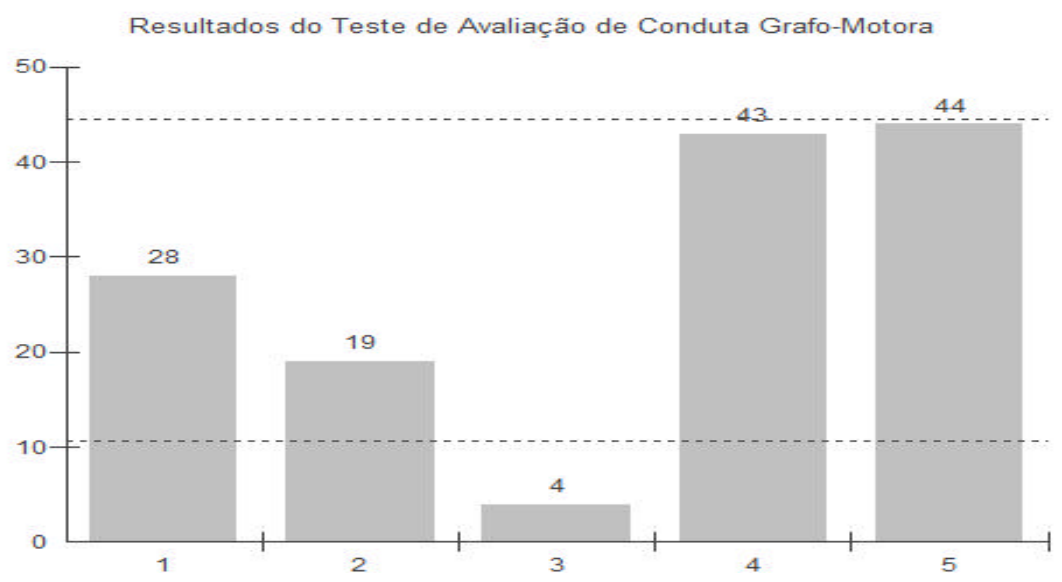

Figura 1 - Resultados do Teste de Avaliação de conduta grafo - motora.

Fonte: dados da pesquisa.

A o se anal isarem esses resultados, chegou-se a al gumas constatações. Uma delas é de que a maioria dos participantes dessa pesquisa foi incapaz de realizar, com êxito, tarefas que exigem empregar a coordenação motora fina o que já era esperado diante do estabelecido na literatura pertinente sobre as sinapses de indivíduos com SD, particularmente, quando se admite que a aprendizagem, em uma de suas interpretações, como nos mostra Rotta, Ohlweiler e Riesgo (2006) e Casarin (2003), pode ser entendida como um processo que se cumpre no sistema nervoso central, no qual se produzem modificações sinaptogênicas, traduzidas por uma modificação funcional ou conductual.

Portanto, reforçando o que dizem autores como Cratty (1979) e Swanson (1990) acerca dos aspectos motores diretamente relacionados ao processo de aquisição da linguagem lecto-escrita, pode-se afirmar que indivíduos com SD apresentam um déficit nas múltiplas relações entre os domínios do comportamento cognitivo e do comportamento motor nomeadamente nas correlações estabelecidas entre a proficiência da leitura e da escrita e nas variáveis de equilíbrio estático, lateralidade, noção do corpo, estruturação espacial e planificação motora.

Quanto ao Teste de Processamento Mental, teve resultados variando entre 1.5 e 7.6 pontos. Os valores que representam a tendência central da amostra são: mediana $=3.8$ e média $=4.4$. Este teste apresentou Coeficiente de Variação $=55.9 \%$.

Os resultados desse teste evidenciaram também que al guns estudantes, como o E3 e E2, tiveram um tempo extremamente lento de reação ao estímulo e, portanto, de emissão de uma resposta ou reação motora, eque esses mesmos estudantes tiveram um baixo escore, 04 e 19 pontos, respectivamente, quando se 
avaliaram variáveis como velocidade, coordenação de agilidade, equilíbrio, coordenação bilateral, força, motricidade global, coordenação dos membros superiores, motricidade composta e motricidade fina, condições necessárias para a aprendizagem e o desenvolvimento da escrita, o que representa um déficit no tempo de reação.

Os resultados da avaliação estão expostos na Figura 2 e possibilitam estabelecer uma correlação entre as variáveis 'estado de atenção' e 'desempenho grafo-motor' em alunos com Síndrome de Down.

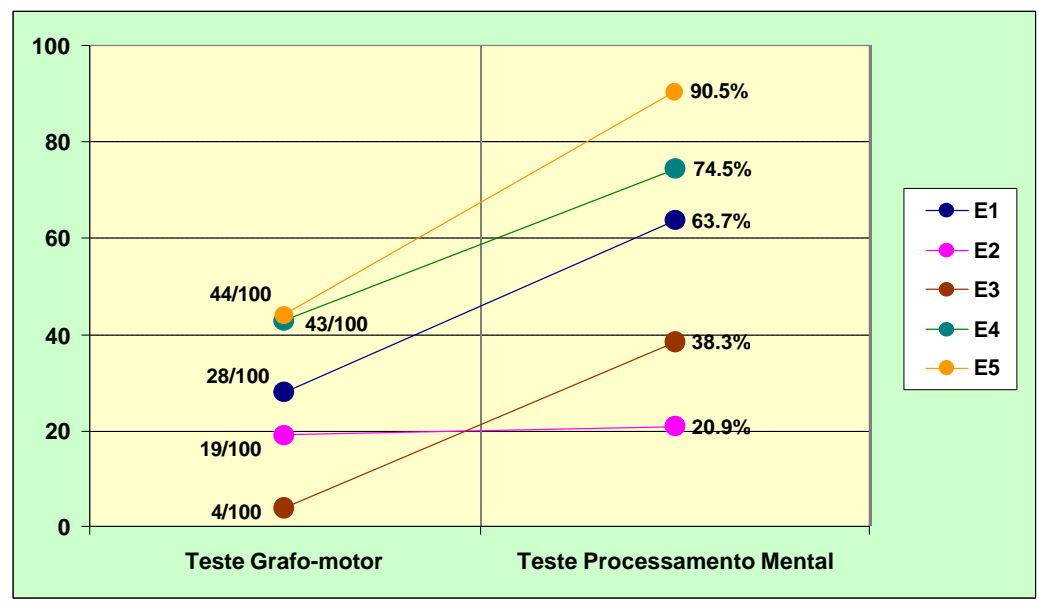

Figura 2 - A valiação da correlação entre o estado de atenção e o desempenho grafo-motor em alunos com Síndrome de Down $(n=5)$.

Fonte: dados da pesquisa

Pôde-se, portanto, visualizar que, para quatro estudantes (80\%), houve correspondência entre os dois testes e somente para um 01 (20\%) o val or dos dois testes manteve-se no mesmo nível, ou seja: o estudante 1 (E1) obteve no teste grafo-motor escore de 28 pontos e um tempo de processamento mental de 63,7; o estudante 2 (E2) foi o único que obteve resultados semelhantes, com 19 pontos para o teste grafo-motor e 20,9 no tempo de processamento mental; o estudante 3 (E3) foi o queteve o menor escore no teste grafo-motor quando obteve 04 pontos, numa escala de 0 a 100, e um tempo de processamento mental de 38,3; o estudante 4(E4) obteve um bom escore para o teste grafo-motor, ao alcançar 43 pontos, tendo um rápido tempo no teste de processamento mental, isto é, 74,5; o estudante 5 (E5) obteve o maior escore no teste grafo-motor, com 44 pontos, portanto, como era de se esperar, teve um bom teste de processamento mental, com um tempo de 90,5 que, como está invertido, para melhor correlação, representa menos de 10 segundos em dez tentativas. 


\section{CONCLUSÃo}

Diante dos resultados obtidos neste estudo, pode-se dizer que as alterações do Sistema N ervoso (SN) da criança com SD, em particular aquelas relacionadas ao déficit de atenção, mantêm relação com o desenvolvimento de condutas grafo-motora necessárias para a aquisição da linguagem escrita em cinco estudantes com SD, de ambos os sexos e idade compreendida entre 17 e 21 anos, participantes do grupo censo deste estudo.

Sendo o desenvolvimento da leitura e da escrita um instrumento fundamental para aquisição de outros conhecimentos e, também, um mecanismo quepermite comunicar nossas vivências, formas de pensar, além denos possibilitar conhecer produções e sentimentos de outras pessoas, concluiu-se que aquela representa um indispensável instrumento de inclusão social, podendo-se inferir que o comprometimento, neste aspecto, poderá afetar as habilidades pertinentes ao processo de aprendizagem de tais adolescentes, habilidades estas, entendidas aqui como um conjunto decondutas e decomportamentos necessários, ou valiosos, para o enfrentamento de demandas interpessoais indispensáveis para o desenvolvimento humano de qualquer indivíduo, seja ele portador ou não de alguma necessidade educativa especial.

Dessa forma, destaca-se a necessidade de se aprofundar no estudo sobre alternativas metodológicas que procurem atuar no déficit de atenção de crianças com SD, para assim, amenizar as dificuldades grafo-motoras, necessárias para a aquisição da linguagem escrita.

Ressalta-se ainda que a intenção ao levantar as dificuldades grafomotora, portanto a dificuldade na aquisição da escrita, em alunos com SD, não é colocar as deficiências na frente do indivíduo, mas, possibilitar o conhecimento de tantas especificidades pertinentes ao assunto, isto porque quando se pretende beneficiar crianças e adolescentes que apresentem necessidades educacionais especiais, torna-se necessário ter conhecimento das possibilidades e limitações dos mesmos, para que se possa criar estratégias coerentes com o seu nível de aprendizagem, oferecendo assim, subsídios para uma intervenção que venham potencializar o desenvolvimento humano de tais entes.

\section{REFERÊNCIAS}

AYRES, M . et al. BioEstat 5: aplicações estatísticas nas áreas das Ciências Biológicas e Médicas. 5. ed. Belém-PA: Publi cações A vul sas do Mami rauá, 2008.

BISSOTO, M.L. Desenvolvimento cognitivoeo processo deaprendizagem do portador deSíndrome deDown: revelando concepções eperspectivas educacionais. Ciências \& Cognição, v. 4, p. 80-88. 2005. Disponível em: বhttp:/ / www.cienciasecognicao.org>. A cesso em: 12 maio 2008.

BRA NDÃ O, M.L. P sicofisiologia. São Paulo: A theneu, 1995.

BRUNONI, D. Aspectos epidemiológicos e genéticos. In: SCHWARTZMAN, J. S. (Org.). Síndrome de D own. São Paulo: Mackenzie. 2003. p. 32-43. 
CASARIN, S. Aspectos psicológicos na Síndrome de Down . In: SCHWARTZMAN, J. S. (Org.) Síndrome de D own. São Paulo: Mackenzie, 2003. p. 263-284.

COCHRAN, G. Tecnologia da amostragem. Rio de Janeiro: Fundo de Cultura, 1956.

CORTESE, S. da S.; MATTOS, P.E.L.; BUENO,J.R. Déficits atentivos eantidepressivos. Jornal Brasileiro de Psiquiatria, Rio de Janeiro, v. 48 n.2, p. 79-85. 1999.

CRATTY, B. Perceptual and motor development. Englewood Cliffs, N ew jersey: Prentice-hall, 1979

DULCAN, M.; BENSON, S. Summary of the practice parameters for the assessment and treatment of children, adolescents, and adults with ADHD. Journal of A merican A cademic Children and A dolescents Psychiatry, v. 36, n. 9, p. 1311-1317, 1997

FLÓREZ, J.; TRON COSO, V.M. Síndrome de D ow n y educacíon. 3. reimp. Barcelona: MassonSalvat Medicina y Santander, 1997.

GOMES, A.M. A criança em desenvolvimento: cérebro, cognição ecomportamento. Rio de Janeiro: Revinter, 2005.

MILLS, N.D. A Educação da criança com Síndrome de Down. In: SCHWARTZMAN, J.S. (Org.) Síndrome deD own. São Paulo: Mackenzie, 2003. p. 232-262.

MOREIRA, L.M .A.; EL-HANI, C.N.;GUSMÃO, F.A.F.A SíndromedeDown esua patogênese: considerações sobreo determinismo genético. Rev. Bras. Psiquiatria, v. 22, n. 2, p. 96-99, 2000. Disponível em: বhttp:/ / www.scielo.br>. Acesso em: 10 maio 2008.

PUESCHE, S. Causas da Síndrome de Down. In: G uia para pais e educadores. São Paulo: Campinas, 2002. p. 53-64.

\section{$\overline{\text { p. } 45-50 .}$}

Panorama histórico. In: . Guia para pais e educadores. São Paulo: Campinas, 2002.

ROSS, A.O. A spectos psicológicos dos distúrbios da aprendizagem e dificuldades na leitura. São Paulo: MacGraw Hill, 1979.

ROTA, N.T.; OHLWEILER, L.; RIESGO, R.S. Transtornos da aprendizagem: abordagem neurobiológica emultidisciplinar. Porto A legre: Artmed, 2006.

SCHMIDT, R.; WRISBERG, C. A prendizagem e performance motora: uma abordagem da aprendizagem baseada no problema. Porto A legre: Artmed, 2001.

SCHWA RTZMA N, J. S. O sistema nervoso na síndrome de Down. In: D own. São Paulo: Mackenzie, 2003. p. 44-81. Síndromede

SILVA, M.F.M.C.; KLEINHANS, A.C.S. Processos cognitivos e plasticidade cerebral na Síndrome de Down. Rev. Bras. Ed. Esp, Marília, v. 12, n.1, p.123-138, 2006. Disponível em: বhttp:/ / www.sciel o.br>. A cesso em: 02 out. 2008.

SILVA, V.F. M etacognição: conteúdo deum segundo cérebro? Laboratório deneuromotricidade. Rio deJaneiro: UCB-RJ, 2004.

SILVA, V; RABELLO R. Especialização Hemisférica; CEMH - Centro de Estudos em Metacognição eH emisfericidade, Rio de Janeiro: UCB-RJ, 2004.

SWANSON, L. H. Executive processing differences beteen learning disabled: mildly retarded and normal achieving children. Journal of A bnormal Child Psychology, v.18, n. 5, p. 549- 563, 1990 WUO, A.S. A construção social da Síndrome de Down. Caderno depsicopedagogia, v. 6, 2007. Disponível em: >http:/ / pepsic.bvs-psi.org.br/ scielo.php?script=sci_arttext\&pid=S1676$492007000100002 \&$ Ing $=$ pt\&nrm=isso >.. A cesso em: 28 out. 2008.

Recebido em: 09/ 04/ 2009

Reformulado em: 30/ 10/ 2009

A provado em: 04/ 11/ 2009 